\title{
A Ferritin-Albumin-Cu Nanoparticle that Efficaciously Delivers Copper (II) Ions to Tumor and Improves the Therapeutic Efficacy of Disulfiram
}

Nuo Xü, Yuan-Fan Yang $\dagger$, Long Chen* and Jian Lin*

N. Xu, Y.-F. Yang, L. Chen, J. Lin

Synthetic and Functional Biomolecules Center, Beijing National Laboratory for

Molecular Sciences, Key Laboratory of Bioorganic Chemistry and Molecular

Engineering of Ministry of Education

College of Chemistry and Molecular Engineering

Innovation Center for Genomics, Peking University, Beijing 100871, China.

L. Chen

E-mail: lcchenlong@163.com

J. Lin

E-mail: linjian@pku.edu.cn

$\dagger$ These authors contributed equally to this work

*Corresponding author 


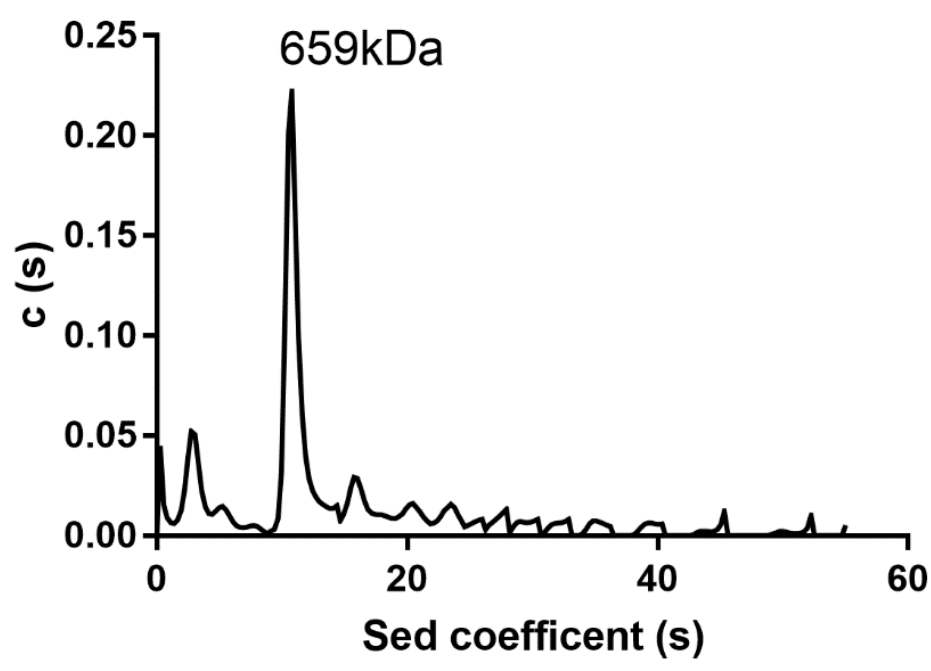

Figure S1. The AUC results of FHC NPs.

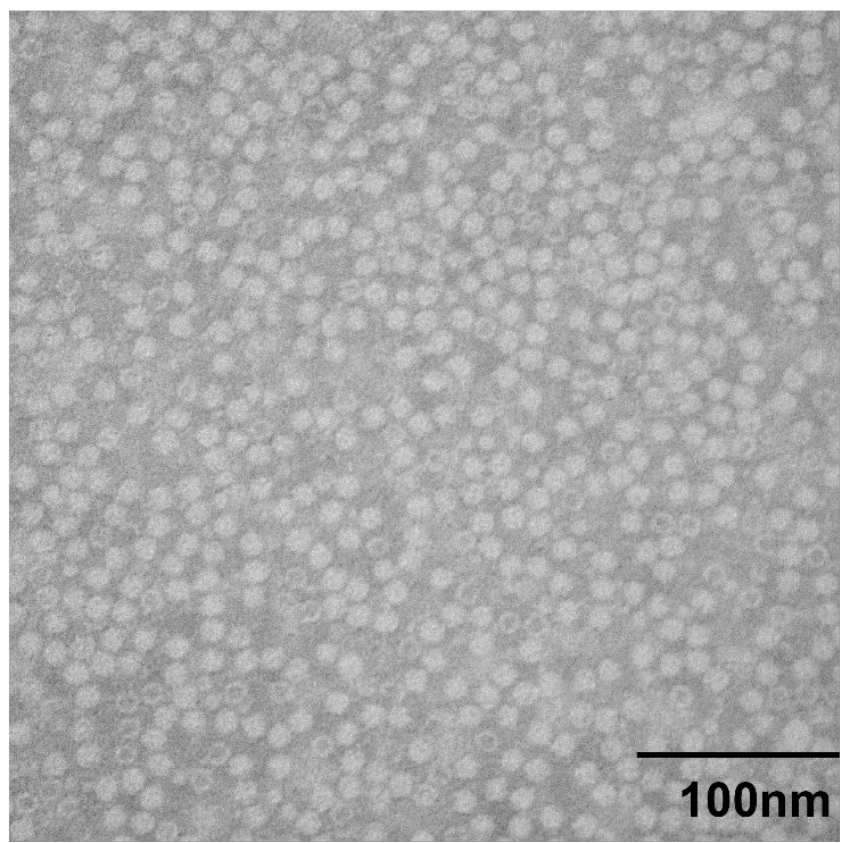

Figure S2. The transmission electron microscopy (TEM) results of ferritin. 


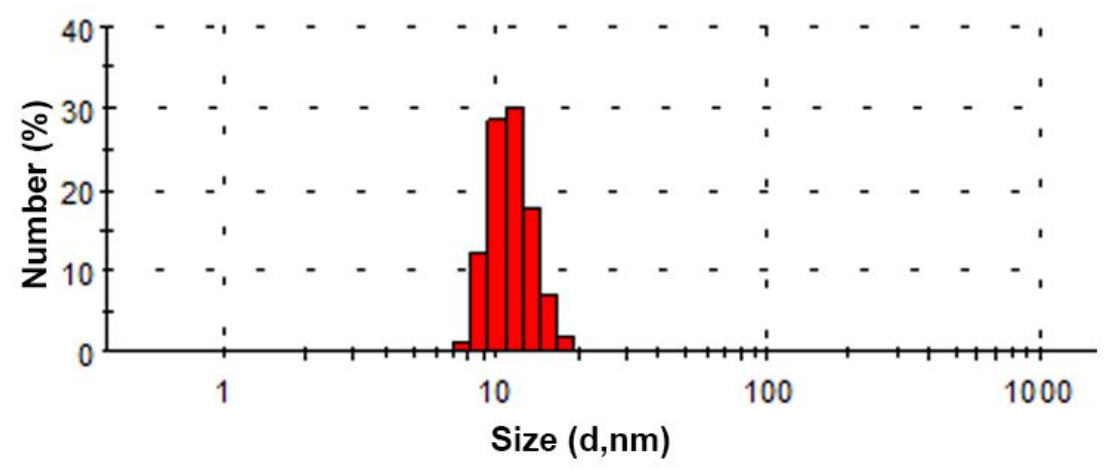

Figure S3. The dynamic light scattering (DLS) analysis of ferritin.

Hoechst 33342

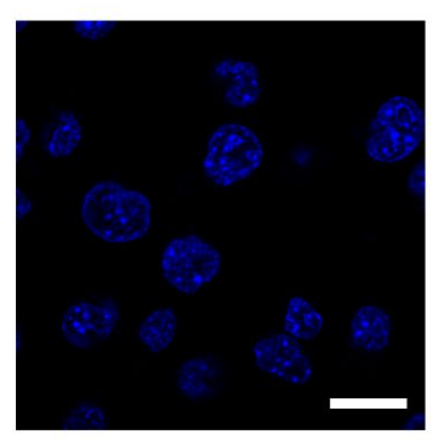

Cy5.5

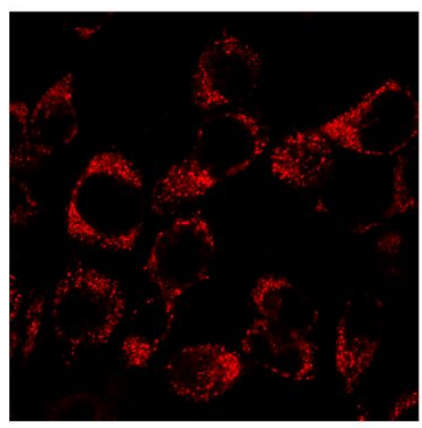

Merge

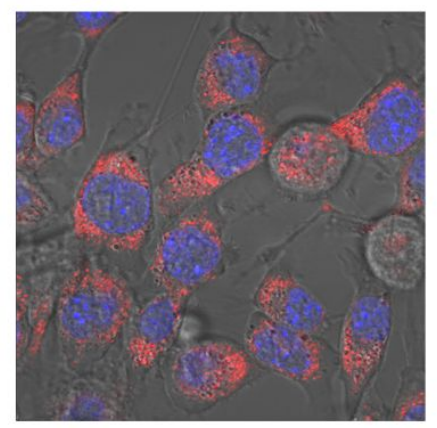

Figure S4. Fluorescence image of Cy5.5 labeled FHC NPs in 4T1 cells. 

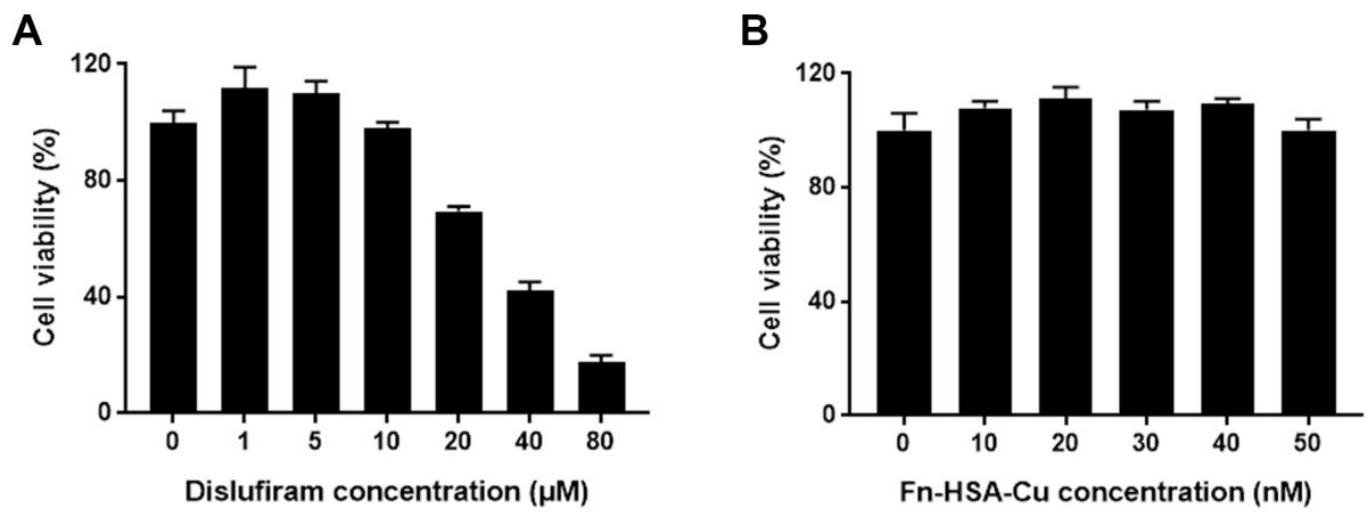

Figure S5. Viability of 4T1 cells treated with different concentration of disulfiram (A) and FHC NPs (B) for $24 \mathrm{~h}$.

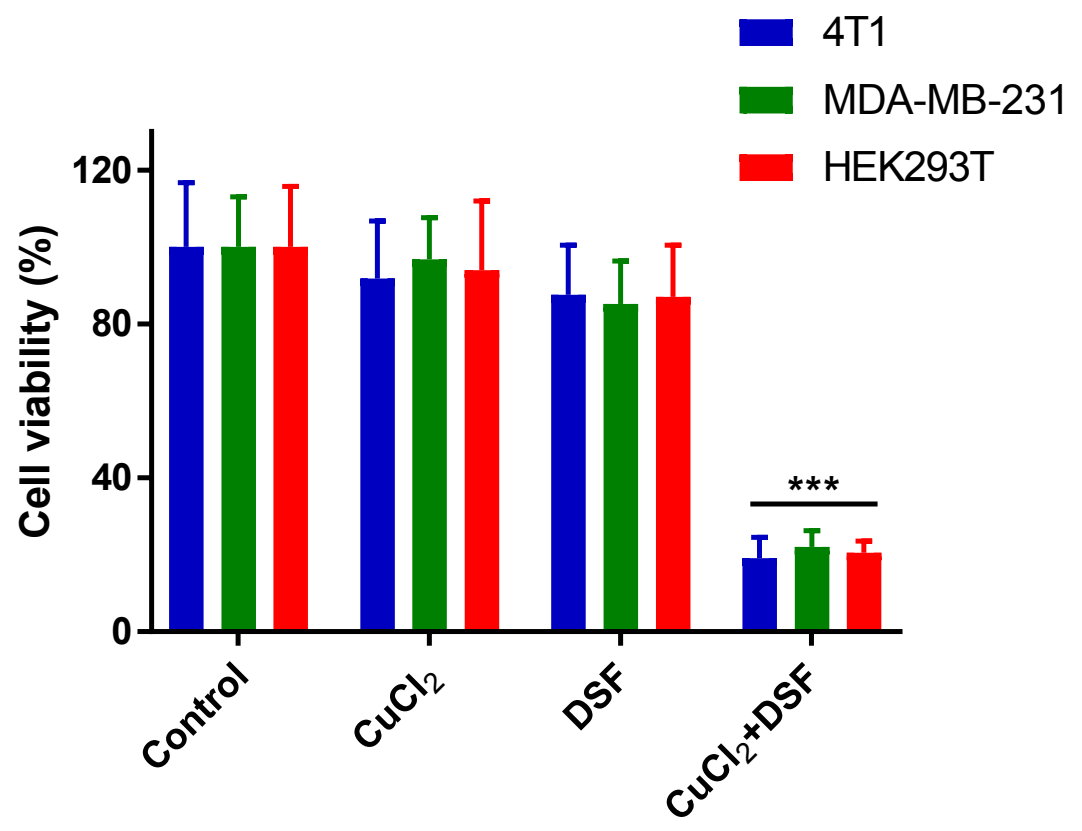

Figure S6. Proliferation inhibition of cancer cells including 4T1 cells, MDA-MB-

231 cells and HEK293T cells treated with $\mathrm{CuCl}_{2}$ and DSF for 24 hours. 
A

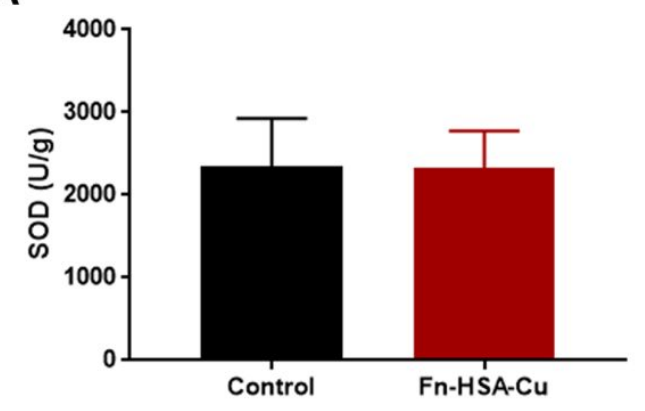

B

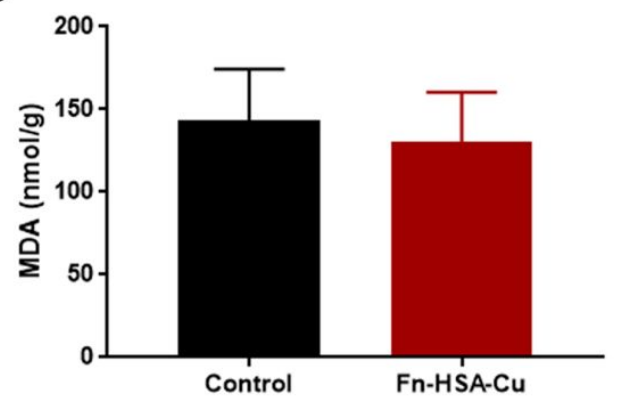

Figure S7. The concentration of SOD (A) and MDA (B) in blood of mice treated with saline and FHC NPs.

Control

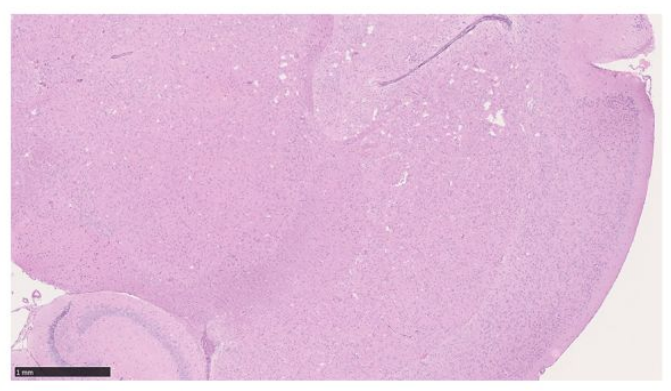

DSF

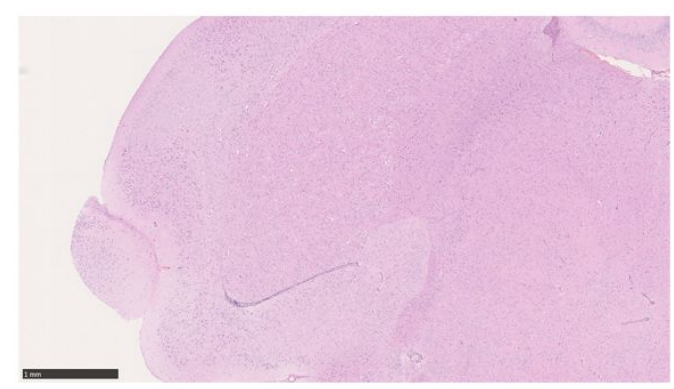

Figure S8. H\&E stained brain tissues collected from mice treated with saline and DSF. 